\title{
INTERACTION MECHANISM OF NON-METALLIC PARTICLES WITH CRYSTALLIZATION FRONT
}

\begin{abstract}
The process of steel solidification in the CCS mould is accompanied by a number of phenomena relating to the formation of non-metallic phase, as well as the mechanism of its interaction with the existing precipitations and the advancing crystallization front. In the solidification process the non-metallic inclusions may be absorbed or repelled by the moving front. As a result a specific distribution of non-metallic inclusions is obtained in the solidified ingot, and their distribution is a consequence of these processes. The interaction of a non-metallic inclusion with the solidification front was analyzed for alumina, for different values of the particle radius. The simulation was performed with the use of own computer program. Each time a balance of forces acting on a particle in its specific position was calculated. On this basis the change of position of alumina particle in relation to the front was defined for a specific radius and original location of the particle with respect to the front.
\end{abstract}

Keywords: non-metallic inclusion, solidification front, steel

\section{Introduction}

During technological processes of steel production nonmetallic inclusions are formed from deoxidation, chemical reactions of liquid metal bath with refractories and entraining of slag into the liquid metal. Generally the presence of non-metallic inclusions deteriorates the quality of material, except the situation when this is done purposefully to produce a specific quantity of non-metallic particles of required chemical composition and size. Continuous steel casting is the last stage where the chemical composition can be corrected. In the solidification process the structure and the distribution of non-metallic inclusions are established in the ingot. The result of distribution of nonmetallic particles in the ingot is a consequence of precipitation processes and segregation of components on the solidification front, and also interactions of non-metallic inclusions with the solidification front. This phenomenon refers to primary particles, i.e. refinery products in the ladle (mainly deoxidation), which were not sufficiently removed from the liquid metal and which moved with the liquid metal to the mould, as well as the secondary precipitates, which were generated while casting. The final distribution of precipitates in the ingot is a consequence of the co-existing processes of precipitation, agglomeration and interaction with the crystallization front [1-5]. These processes are responsible for a non-homogeneous distribution of non-metallic inclusions, which mainly manifests itself in the concentration of contaminants in the central part of the ingot.

The density of majority of non-metallic inclusions is lower than liquid steel density, that is why they flow out of liquid steel.
During ascending they collide, as a consequence of collisions larger agglomerates may be formed. The process in which inclusions flow out in the CCS mould is favored by the introduction of argon, or magnetic mixing. With the increase of the casting rate, especially when thin slab casting, the removal of inclusions is more difficult. Therefore the conditions in which they are removed in the solidification front area are responsible for the final quality of the cast products.

The analysis of interaction of a group of particles with the crystallization front is difficult and requires looking at the distribution of forces acting on a particle in the front area and also solving the population balance equation, analyzing the phenomenon of particles agglomeration and flotation. A particle may be present in an area where two crystallization fronts interact simultaneously. If we assume a dendritic or cellular type of front, the solving of this problem for a group of particles will be very difficult. In the case of a dendritic type of front the particle may be trapped between dendritic arms, absorbed by crystallization front or repelled. If the diameter of the inclusion is smaller than the distance between dendritic arms $d_{p}<P D A S$ (Primary Dendrite Arm Spacing), the particle is trapped in the interdendritic space. If the diameter of the particles $d_{p}>P D A S$, the inclusion may be absorbed or repelled by the front [6]. The repelling or absorption is conditioned by local cooling conditions, flow of liquid steel and density of particles. These conditions stem from the equilibrium of forces acting on a particle when it occupies different positions with respect to the front. A direct observation of the behavior of non-metallic particles in liquid metal is possible only on its surface. Their behavior could be analyzed 
mainly on the basis of low melting temperature model liquids. Numerous authors performed simulations and experiments on the behavior of non-metallic particles during the process taking place in CCS [1-2,6-12]. A CCD [4] or digital camera, or a laser microscope was used for experimental analyses of the particle/ front interaction [13].

Authors of this paper analyzed the behavior of a single non-metallic particle $\mathrm{j}$ limiting only to its interaction with a flat solidification front for different locations with respect to the front, accounting for the local gradient of liquid steel flow rate.

\section{Interaction of non-metallic particle with vertical solidification front}

In the case of a flat crystallization front the interaction of the crystallization front with non-metallic particle $\mathrm{j}$ lies in the absorption or repelling of the particle. The main criterion deciding whether or not a non-metallic particle is absorbed by the front at small crystallization rates, is the free energy of absorption [14]:

$$
\Delta G_{p .}=\sigma_{I-S}-\sigma_{I-L} \leq 0
$$

where:

$$
\begin{gathered}
\sigma_{I-S}-\text { surface energy (precipitate }- \text { solid metal) }[\mathrm{N} / \mathrm{m}], \\
\sigma_{I-l}-\text { surface energy (precipitate }- \text { liquid metal) }[\mathrm{N} / \mathrm{m}] .
\end{gathered}
$$

The particles are absorbed by the front when the result is negative. In the case of high crystallization rates the particles are absorbed in any case. The experimental data on the critical rate of solidification are very poor and refer only to particles on the surface of liquid metal in the moving solidification front. In the case of $\mathrm{Al}_{2} \mathrm{O}_{3}$ particles in steel, Shibata et al. [15] stated that the critical rate of the crystallization front is expressed by the formula:

$$
V_{k r}=\frac{60}{r}(r \text { in } \mu \mathrm{m})
$$

which gives $1.2 \mu \mathrm{m} \cdot \mathrm{s}^{-1}$ for particles of radius $50 \mu \mathrm{m}$.

- Stefanescu et al. [16] observed that for particles $\mathrm{SiC}$ in aluminum alloys with small admixture of magnesium or nickel $V_{k r}$ values are critical for particles of radius $50 \mu \mathrm{m}$ and stay within a broad range of $8-400 \mu \mathrm{m} \cdot \mathrm{s}^{-1}$.

- Physically this phenomenon describes a system of forces acting on a particle and from which we have a velocity vector. In a situation corresponding to the conditions of CCS mould, the following system of forces acts on a particle [17]:

- force of gravity:

$$
F_{g}=\frac{4}{3} \cdot \pi \cdot r^{3}\left(\rho_{m}-\rho_{c z}\right) \cdot g
$$

where:

$F_{g}$ - difference of buoyancy and gravity force [N],

$\rho_{m}-$ density of metal (steel) $\left[\mathrm{kg} / \mathrm{m}^{3}\right]$,

$\rho_{c z}-$ density of particles (inclusions) $\left[\mathrm{kg} / \mathrm{m}^{3}\right]$,

$g-$ acceleration of gravity $\left[\mathrm{m} / \mathrm{s}^{2}\right]$.
For vertical solidification fronts we cannot assume that the particle is immobile. The movement of particles with respect to the surrounding fluid and the front should be assumed. A flat system of forces acting on the particle encompasses the force of gravity expressed by equation (3). The viscous resistance force of opposite direction to the direction of particles with velocity $V_{p}$ as compared to liquid, is presented in the form [7]:

$$
F_{d}=6 \cdot \pi \cdot \mu \cdot r \cdot V_{p} \cdot \theta
$$

$F_{d}-$ viscous resistance force $[\mathrm{N}]$,

$\mu-$ coefficient of dynamic viscosity $[\mathrm{kg} / \mathrm{m} \cdot \mathrm{s}]$,

$V p$ - flow rate of particles with respect to fluid [m/s].

Coefficient $\theta$ depends on the direction of the particle and its distance from the front $h$ :

- for particles distant from the front: $\theta=1$,

- for particles approaching the front: $\theta=\frac{r}{h}$,

- for particles moving parallel to the front: $\theta=\ln \left(\frac{r}{h}\right)$.

In the vicinity of the solidification front the fluid flow rate is non-homogeneous (the front-parallel component is important) and the concentration of fluid components is non-homogeneous, especially those, which have low equilibrium partition coefficient $k^{s / 1}$

The force produced by the gradient of fluid flow rate $S$ perpendicular to the front surface occurs in the area where it can be identified as a boundary speed layer, is called the Saffman force [17].

- Saffman force:

$$
F_{S}=6.46 \cdot \mu \cdot r^{2} \cdot V_{c z} \cdot \sqrt{\frac{S}{v}}
$$

where:

$F_{s}-$ Saffmann force [N],

$v$ - kinematic viscosity $\left[\mathrm{m}^{2} / \mathrm{s}\right]$,

$S$ - local gradient of fluid rate $[1 / \mathrm{s}]$.

This force may cause approaching or drifting apart of particles from the front, depending on the direction of the fluid and difference of density of the particle and fluid. If the fluid flows down along the front and the density of the particle is bigger than that of the fluid, the Saffman lift moves away the particle from the front. A schematic of force equilibrium for this case was presented in Fig. 1. The repulsive force $F r$ starts acting only when the particle approaches the front to a distance comparable to the interatomic distances.

- Non-homogeneity of concentration of a surfactant (e.g. sulphur or oxygen) in the neighborhood of the crystallization front (in the boundary concentration layer) activates the force which pushes the particle to the front surface. This can be classified as Marangoni effect. Mukai and Zeze [8] made a convincing experiment for water solutions, in which this phenomenon evidently took place in the presence of surfactants. Force $F_{M}$ acting on a particle is a function of gradient of interphase energy $\sigma_{I-l}$. 
- Mukai force:

$$
F_{M}=-\frac{8}{3} \cdot \pi \cdot r^{2} \cdot \frac{\partial \sigma_{I-l}}{\partial z}
$$

where:

$F_{M}$ - force evoked by local gradient of surface energy [N], $z$ - horizontal coefficient,

- $\quad$ The resulting speed with which particles move is also proportional to the gradient of interphase energy. The liquid metal flow has an influence on the interaction between particles and solidification front. The gradient of fluid rate near the solidification front is approximately given by equation:

$$
S=\frac{4 \cdot V_{0}}{h}
$$

$h$ - distance of particles from vertical solidification front [m],

$V_{0}-$ average flow rate of steel (downwards) [m/s].

The flow of critical rate for given parameters of the front and particles completely stops the absorption of particles. For bigger particles of higher density the higher critical rate of the front is needed, so that the particle is not absorbed. The same applies to the shape of the front and crystallization rate. For higher coarseness of the front and higher crystallization rate the higher critical flow rate is needed to stop the absorption.

\section{Numerical model for particle movement near to crystallization front}

In this work $\mathrm{Al}_{2} \mathrm{O}_{3}$ particle movement in the liquid steel is analysed. It is assumed that particle has uniform density and its shape can be approximated with sphere of $r$ radius, which will be in the following part named substitute radius. It is assumed that liquid velocity gradient is known. Particle movement near to crystallization front may be described with the following mathematical model:

$$
\begin{gathered}
a=\frac{\left|F_{w}\left(F_{S}, F_{g}, F_{d}, v\right)\right|}{4 / 3 \pi r^{3} \rho_{c z}} \\
\left\{\begin{array}{l}
\frac{d v_{x}}{d t}=a_{x}, \\
v_{x}(t=0)=v_{x}^{0}
\end{array}\right. \\
\left\{\begin{array}{l}
\frac{d v_{y}}{d t}=a_{y}, \\
v_{y}(t=0)=v_{y}^{0}
\end{array}\right.
\end{gathered}
$$

where:

$A$ - vector of particle acceleration, $a_{x}$ - acceleration vector component in $x$ axis direction, $a_{y}$-acceleration vector component in $y$ axis direction, $v$ - vector of particle speed, $v_{x}-$ speed vector component in $x$ axis direction, $v_{y}$ - speed vector component in $y$ axis direction, $v_{x}{ }^{0}, v_{y}{ }^{0}$-initial values of speed, which takes into account fluid gradient, $r$ - substitute particle radius, $\rho_{c z}$ - particle density, $F_{w}$ - resultant force vector, $F_{S}-$ Saffmann force vector, $F_{g}$ - buoyancy force vector, $F_{d}$ - liquid resistance force. Result of the modeling is location of the particle at each and every moment in time: $(x(t), y(t))$, which can be described with following set of ordinary differential equations:

$$
\begin{aligned}
& \left\{\begin{array}{l}
\frac{d x}{d t}=v_{x}, \\
x(t=0)=x^{0}
\end{array}\right. \\
& \left\{\begin{array}{l}
\frac{d y}{d t}=v_{y}, \\
y(t=0)=y^{0}
\end{array}\right.
\end{aligned}
$$

where:

$x^{0}, y^{0}-$ is the initial location of the particle, $x, y, v_{x}, v_{y}$-are variables dependent on the time $t$.

To define vectors components in $x$ and $y$ axis direction trigonometric functions for resultant $F_{w}$ force are calculated:

$$
\begin{gathered}
\left|F_{w}\right|=\sqrt{\left|F_{g}\right|^{2}+\left|F_{d}{ }^{2}\right|} \\
\sin \alpha=\frac{\left|F_{g}\right|}{\left|F_{w}\right|}=\frac{\left|F_{g}\right|}{\sqrt{\left|F_{g}\right|^{2}+\left|F_{d}\right|^{2}}} \\
\cos \alpha=\frac{\left|F_{S}\right|}{\left|F_{w}\right|}=\frac{\left|F_{S}\right|}{\sqrt{\left|F_{g}\right|^{2}+\left|F_{d}\right|^{2}}}
\end{gathered}
$$

Liquid in analysed domain can flow relatively to the crystallization front. Its flowing speed can be described in $x$ and $y$ separately. Those values affect particle movement. At the stage of numerical solving the liquid movement speed components are added to initial values of particle speed components. To solve set of ordinary differential equations presented above (eqs 2, 3) Euler's forward scheme witch constant time step $\Delta t$ is applied. The aim of the computations is to create the numerical sequence of following values:

$$
\left(t^{n}, x^{n}, y^{n}, v_{x}^{n}, v_{y}^{n}\right), n=1,2,3, \ldots
$$

which values are approximate positions and particle speeds at equidistant time steps. Consecutive values are given with following iterative formula:

$$
\begin{gathered}
x^{n+1}=x^{n}+\Delta t v_{x}^{n} \\
y^{n+1}=y^{n}+\Delta t v_{y}^{n} \\
v_{x}^{n+1}=v_{x}^{n}+\Delta t a_{x} \\
v_{y}^{n+1}=v_{y}^{n}+\Delta t a_{y} \\
t^{n+1}=t^{n}+\Delta t, t^{0}=0
\end{gathered}
$$


After each and every time step the acceleration $a$ value is updated as its value depends on resultant force vector (eq. 1) and its components are calculated on the base of trigonometric functions (eq. 5 and 6) values:

$$
\begin{aligned}
& a_{x}=a \cos \alpha \\
& a_{y}=a \sin \alpha
\end{aligned}
$$

Base on the numerical model given above computational procedures were prepared and the original software was written.

\section{Results and discussion}

Simulation was performed with the use of own computer program that base on equations for forces acting on a particle (Eqs. 3-5) as well as a mathematical and numerical model (Eqs 8-23). The calculation of the trajectory of the particle in the vicinity of a flat vertical solidification front was performed for $\mathrm{Al}_{2} \mathrm{O}_{3}$ - a product of deoxidation. The following parameters were assumed for steel and the particle (Table 1):

TABLE 1

Data referring to materials and calculation parameters [1]

\begin{tabular}{|l|c|}
\hline \hline steel density & $7000 \mathrm{~kg} / \mathrm{m}^{2}$ \\
\hline particle density & $3990 \mathrm{~kg} / \mathrm{m}^{2}$ \\
\hline dynamic viscosity & $0.06 \mathrm{~m}^{2} / \mathrm{s}$ \\
\hline time step & $110^{-6} \mathrm{~s}$ \\
\hline local velocity gradient & $0.0001 ; 0.00001 ; 0.000001$ \\
\hline particle radius & $10-100 \mu \mathrm{m}$ \\
\hline
\end{tabular}

The calculations were carried out for three different locations of inclusions in reference to the front, i.e. for a particle at a distant position, moving parallel to the front and in a close proximity. The interaction was analyzed for particles of radius 10 , 20, 25 and $100 \mu \mathrm{m}$, and each of them for various gradients of speed (Figs. 1-9). The same calculation procedure was applied for cycles of constant duration equal to $100 \mathrm{~s}$. Characteristically the particles were observed to move up, which is a consequence of the acting force of gravity due to density of the inclusion and the steel matrix difference. The first series was performed for a particle at a distant position in respect to the solidification front (Figs. 1-3). It was assumed that the local gradient of velocity had an influence on the trajectory of the particle so it was taken into account during the calculations. At a high gradient of velocity the particle was pushed towards the front, at a low and moderate gradient the flotation process dominated and the interaction between the particle and the front was negligible. A high gradient made the particle approach the crystallization front, and this trend was maintained both for small and large inclusions.

Analogous simulations were performed for a particle moving parallel to the front. The results for inclusions of radius 20, 25 and $100 \mu \mathrm{m}$ have been presented in (Figs. 4-6).

The gradient of velocity was observed to have a decisive influence on the trajectory of the particle, causing its floating

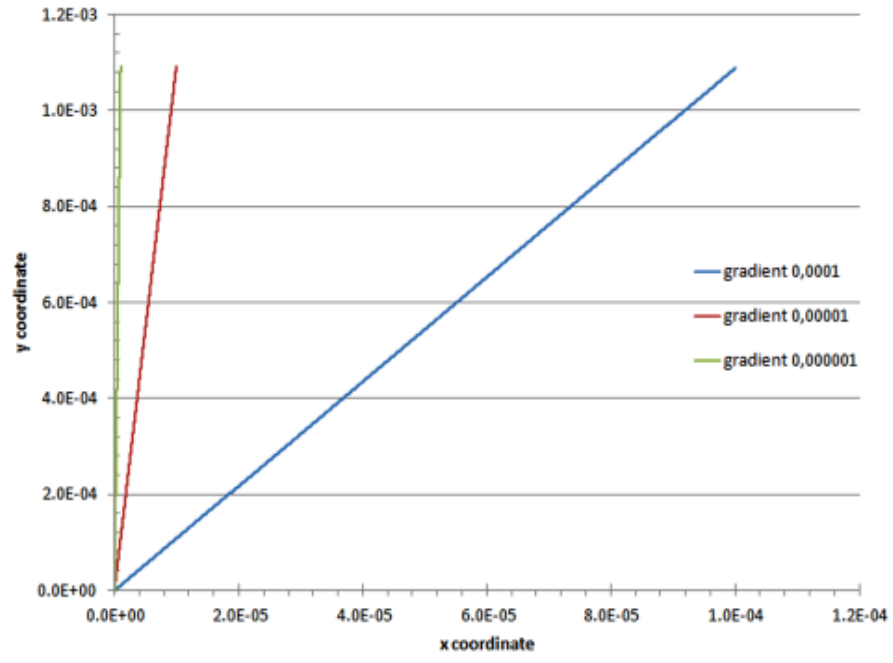

Fig. 1. Influence of local gradient of velocity on the movement of particle of radius $10 \mu \mathrm{m}$

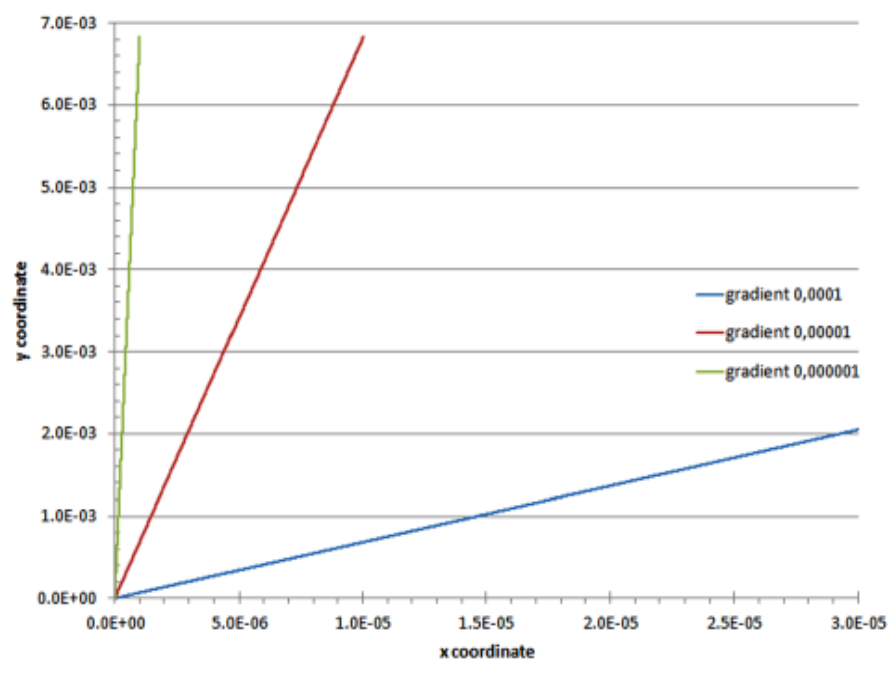

Fig. 2. Influence of local gradient of velocity on the movement of particle of radius $25 \mu \mathrm{m}$

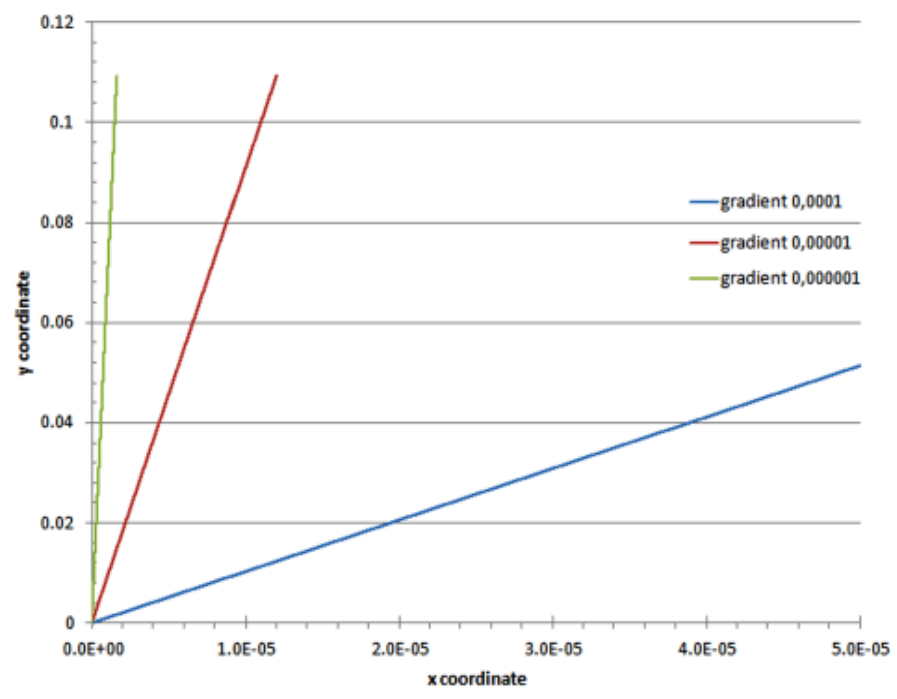

Fig. 3. Influence of local gradient of velocity on the movement of particle of radius $100 \mu \mathrm{m}$ 


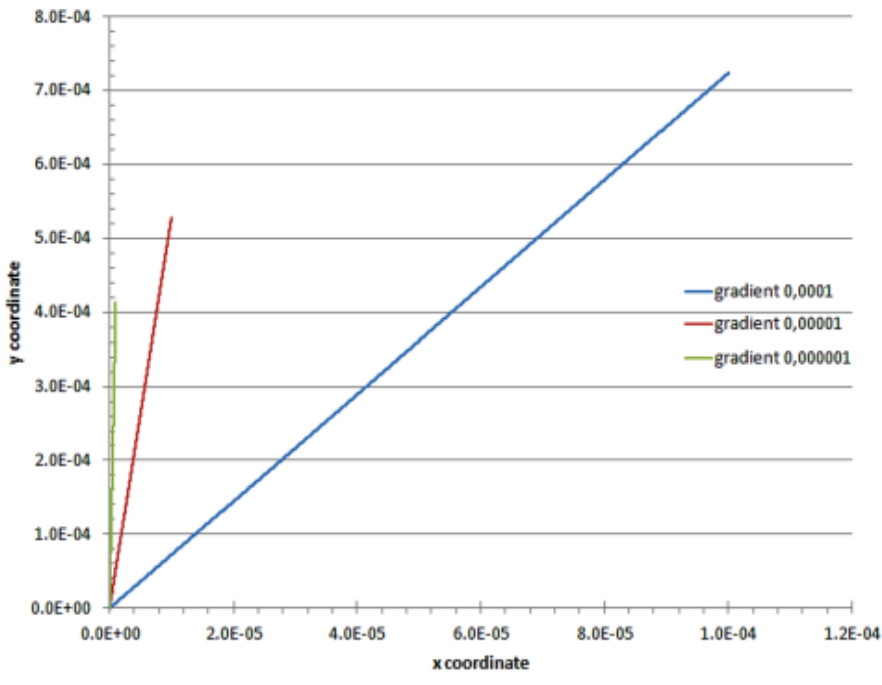

Fig. 4. Influence of local gradient of velocity on the movement of particle of radius $20 \mu \mathrm{m}$

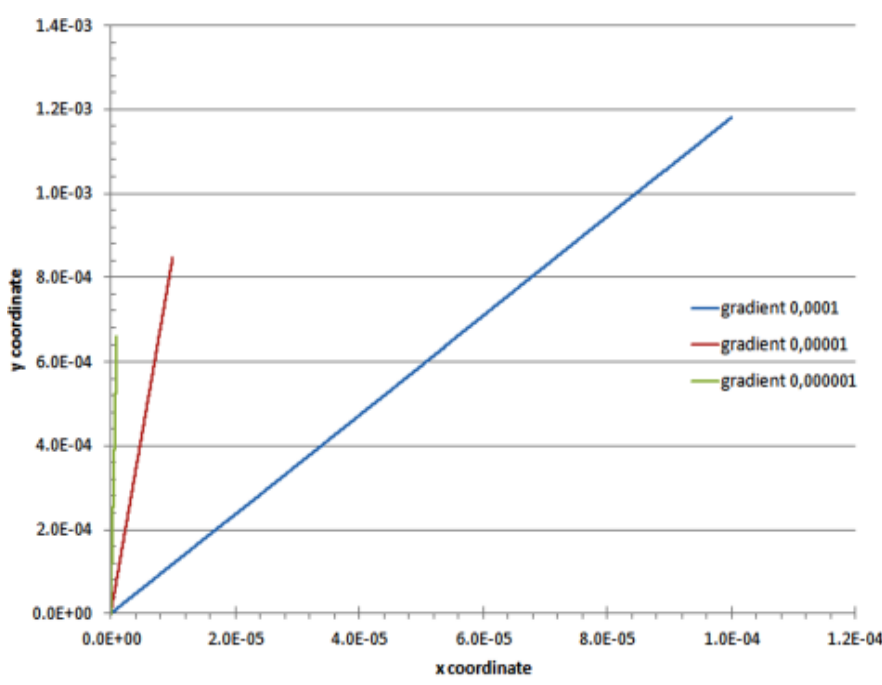

Fig. 5. Influence of local gradient of velocity on the movement of particle of radius $25 \mu \mathrm{m}$

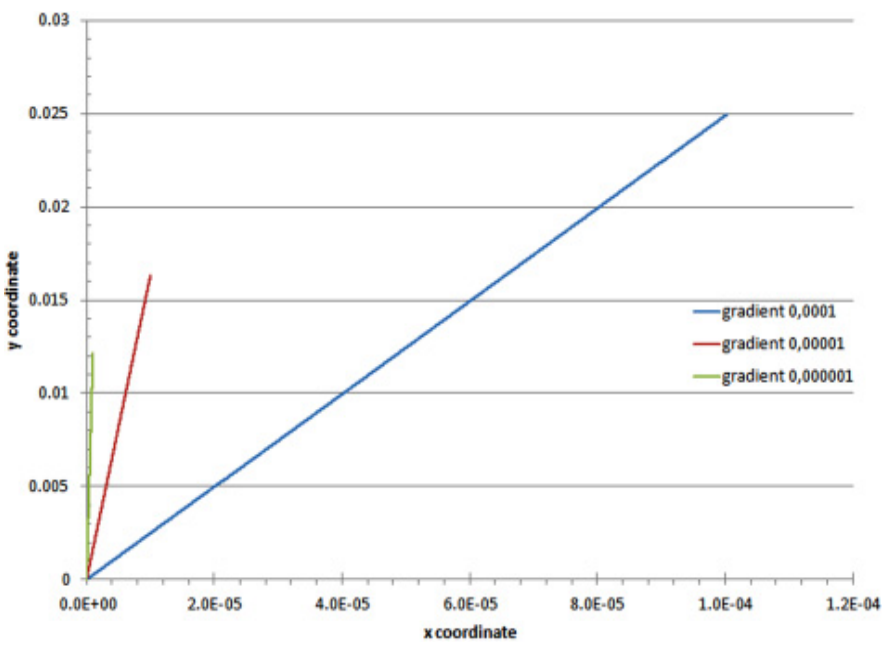

Fig. 6. Influence of local gradient of velocity on the movement of particle of radius $100 \mu \mathrm{m}$ and simultaneous approaching the front. In the case of high gradient $(0.0001)$ the particle was pushed towards the front, no matter the size of the particle. Characteristically, particles of different size reached the same position in respect to the starting point for a given gradient. At a low gradient the particles moved vertically up; analogously to variant 1 particles of large radius floated faster.

The third series of calculations was performed for a particle moving towards the front. The effect evoked by the repulsive force, whose range covered an area of atomic distance, has been neglected. The Mukai effect has not been taken into account either.

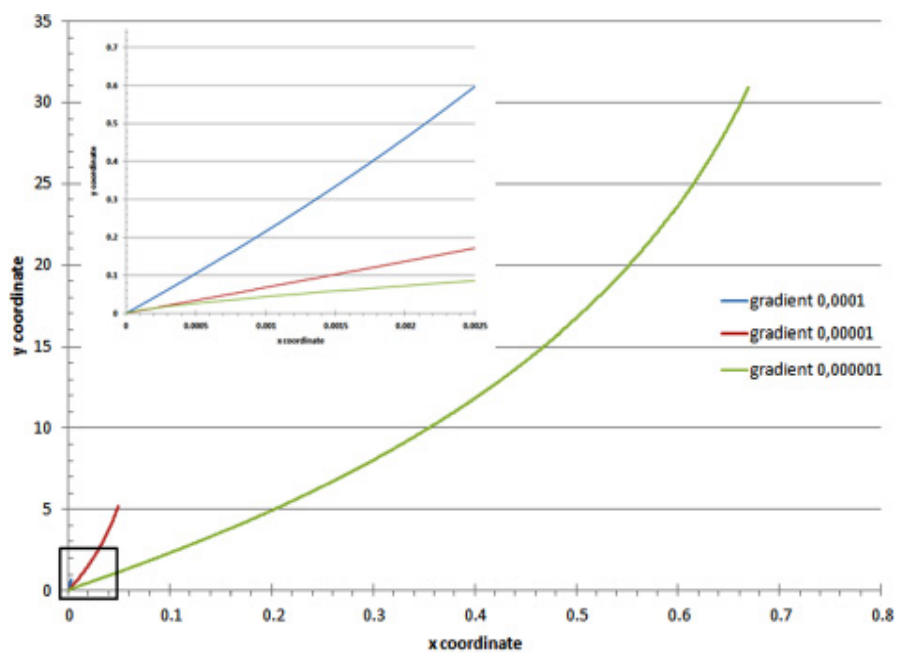

Fig. 7. Influence of local gradient of velocity on the movement of particle of radius $10 \mu \mathrm{m}$

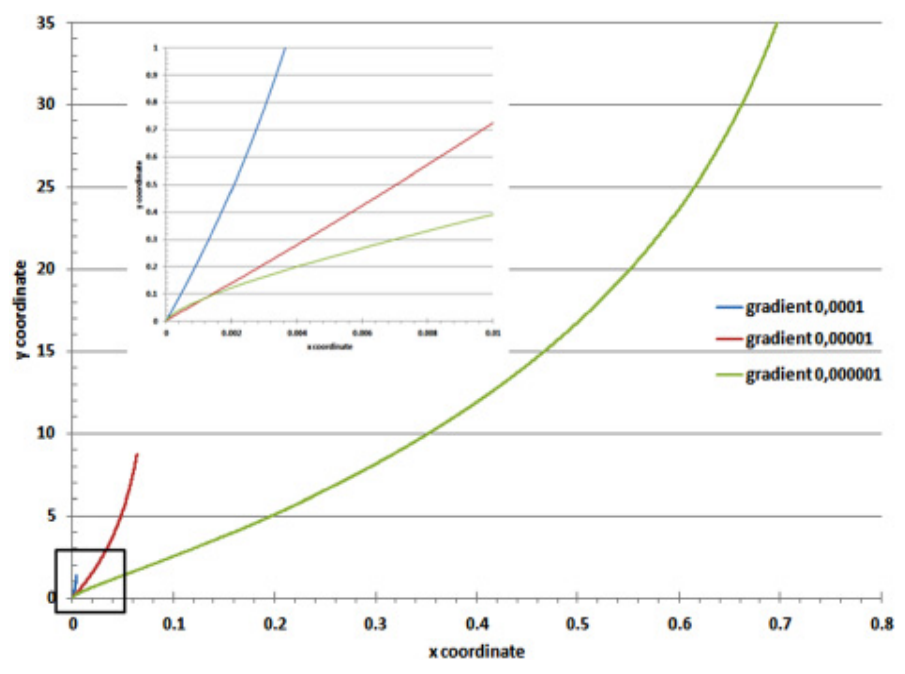

Fig. 8. Influence of local gradient of velocity on the movement of particle of radius $25 \mu \mathrm{m}$

Particles of all size moved towards the front and this tendency was particularly well visible for low gradients of velocity. At a high and moderate gradient the particles were observed to float without any interaction with the front. A low gradient made small and large particles approach the crystallization front. For 


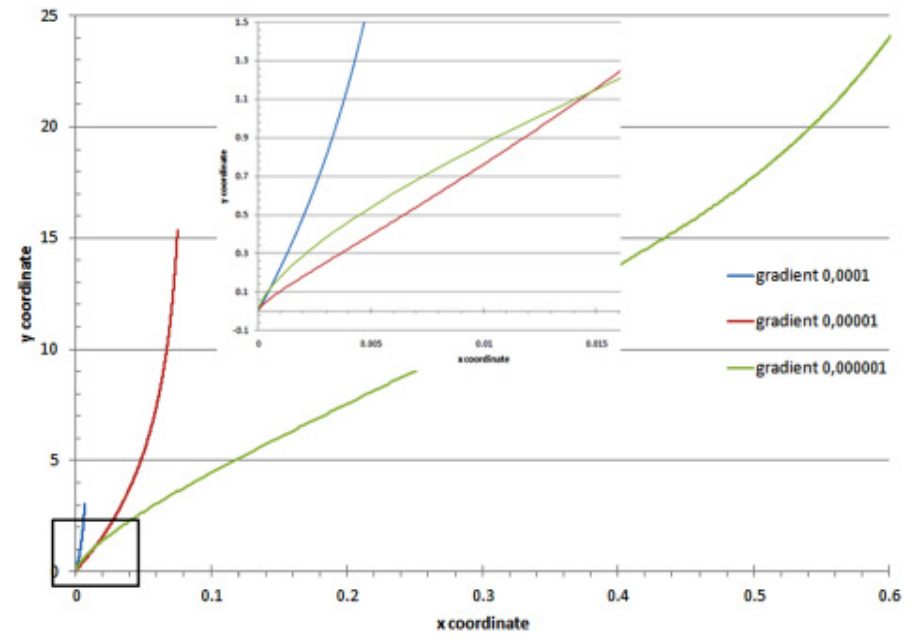

Fig. 9. Influence of local gradient of velocity on the movement of particle of radius $100 \mu \mathrm{m}$

a particle of radius $10 \mu \mathrm{m}$ the approaching tendency was observed to weaken after some time on behalf of flotation, which started to dominate. Analogous mechanisms were noted for a particle of radius $20 \mu \mathrm{m}$. No pushing mechanisms were observed for large particles. Interestingly, at an early stage all particles were pushed towards the front to a certain moment. This process depended both on the gradient and on the size of the particle.

\section{Conclusion}

The analysis of movement of a particle in the neighborhood of the solidification front allowed authors to distinguish a few cases of interaction, not as previously assumed, two extreme situations, i.e. attraction and pushing away. In the case of particles placed at a considerable distance from the front the Stokes mechanism dominated and the velocity of floating of particles did not depend on the gradient of velocity. The pushing of particles towards the front was applicable mainly to large gradients of velocity. A certain analogy was observed for variant II, i.e. when the particle moved parallel to the front. The pushing of particles towards the front was observed only for high gradients; however the flotation of inclusions, especially at a very small gradient of velocity, was considerably smaller. The most complex case was the interaction of particles moving towards the front. In this case the high gradient did not favor assimilation of particles by the solidification front, especially in reference to small inclusions.

\section{Acknowledgement}

This work was sponsored by Ministry of Science as the statute work AGH UST - University of cience and Technology in Krakow (contract 11.11.170.318.14)

\section{REFERENCES}

[1] D. Kalisz, Termodynamiczna charakterystyka powstawania fazy niemetalicznej w ciekłej stali, Akapit Krakow (2013).

[2] D. Kalisz, Archives of Metallurgy and Materials 59, 2, 493-500 (2014).

[3] D. Kalisz, P. L. Żak, Kovove Materialy 53, 35-41 (2015).

[4] P.L. Żak, D. Kalisz, J. Lelito, M. Szucki, B. Gracz, J.S. Suchy, Metallurgy 54, 2, 357-360 (2015).

[5] S. Kimura, Y. Nabeshima, K. Nakajima, S. Mizoguchi, Metal Trans. B 31 B, 1013-1021 (2000).

[6] C. Pfeiler, B.G. Thomas, M. Wu, A. Ludwig, A. Kharicha, Steel Research Int. 77, 7, 1-10 (2006).

[7] Q. Han, J. Hunt, ISIJ Int. 35, 693-699 (1995).

[8] K. Mukai, M. Zeze, Steel Research 74, 3, 131-138 (2003).

[9] L. Zhang, JOM. 65, 9, 1138- 1144 (2013).

[10] J. Rogge, J.Crystal Growth, 94, 726-738 (1989).

[11] S. Sen, B.K. Dhindaw, D.M. Stefanescu, A. Catalina, P.A. Curreri, J. Crystal Growth 173, 574-584 (1997).

[12] D. Shangguan, S. Ahuja, D.M. Stefanescu, Met. Trans. A 23A, 669-680 (1992).

[13] H. Esaka, Y. Kuroda, K. Shinozuka, M. Tamura, ISIJ Int. 44, 682 (2004).

[14] S.N. Omenyi, A.W Neumann, J.Appl. Phys., 47, 9, 3956-3962 (1976).

[15] H. Shibata, H. Yin, S. Yoshinaga, T. Emi, M. Suzuki, ISIJ Int., 38, 149-156 (1998).

[16] D.M. Stefanescu, B.K. Dhindaw, S.A. Kacar, A.Moitra, Met. Trans. A 19A, 2847-2855 (1988).

[17] P.G. Saffman, J. Fluid Mech. 22, 385-400, (1965). 